\title{
Appendicitis as a cause of small bowel obstruction in pediatric age group
}

\author{
Sharma Mitrajit ${ }^{1}$, Lahiri Kaushik ${ }^{2}$
}

Received on August 22, 2018; editorial approval on December 09, 2018

\begin{abstract}
Intestinal obstruction is a common surgical emergency in children caused by varied conditions. Although bowel paralysis accompanying acute appendicitis is well known, obstruction of large or small intestine caused by appendicitis is extremely rare with very few cases reported in literature. The diagnosis of such a condition is possible only on table. Every emergency surgeon needs to be aware of such a possibility. We report a case of a 6-year-old male presenting with classical features of intestinal obstruction for 4 days with no history and clinical finding suggestive of an episode of appendicitis. On laparotomy small bowel obstruction was seen and appendix was found to be the cause. We reviewed literature to find similar cases reported in the past.
\end{abstract}

Keywords: Right iliac fossa tenderness; intestinal obstruction; laparotomy.

\section{INTRODUCTION}

Intestinal obstruction is one of the common causes of acute abdomen in children. Although appendicitis causing intestinal obstruction is a rare cause with only a few reported cases in literature, among the cases there has been only one report of this occurrence in pediatric age group. ${ }^{1}$ Mechanical obstruction, with or without strangulation, can result from loops of the small bowel becoming entangled and pinched by the inflamed appendix, or adhesion of the distal end of the appendix to loops of the small bowel, caecum, or retroperitoneum. ${ }^{2}$ Here we report a case where the inflamed appendix caused a band like structure resulting in intestinal obstruction.

\section{Case History}

A 6-year-old male child presented with abdominal pain with vomitting for 4 days with constipation for 3 days. Abdomen was distended with tenderness elicited on the palpation of lower abdomen. Per rectal examination was done to rule out fecal impaction or mass and was found to be normal.

$\mathrm{X}$-ray plain picture erect abdomen showed dilated bowel loops suggestive of bowel obstruction. A diagnosis of mechanical intestinal obstruction of unknown etiology was made and was scheduled for emergency laparotomy. The abdomen was then opened by an upper right transverse incision. Dilated bowel loops were identified and inflamed appendix was seen lying over the distal ileum and tip was attached to the ileocecal mesentry resulting in a band like structure causing obstruction. Band was released followed by appendicectomy. Post-

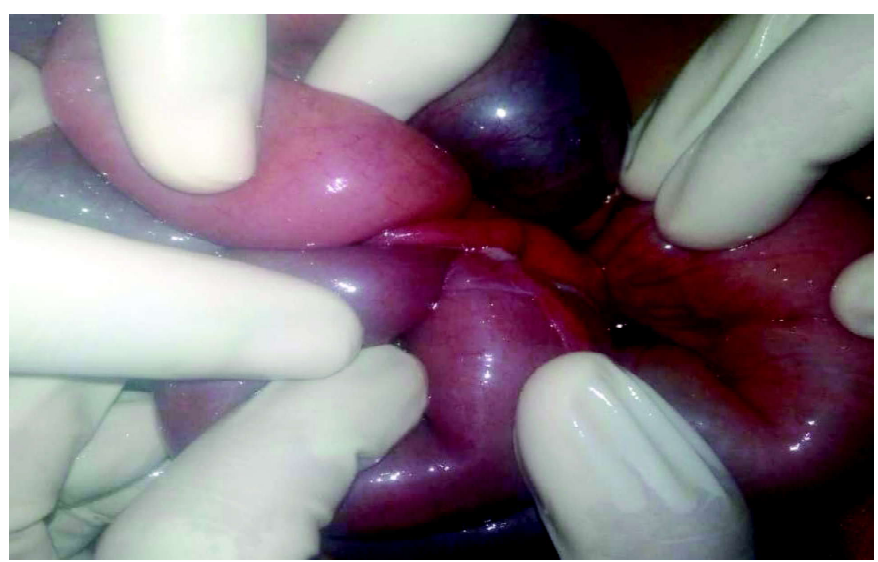

Figure 1 On table picture showing the appendix acting as an obstruction band over distal ileum

\section{Address for correspondence:}

${ }^{1}$ Post Graduate Trainee (Corresponding author)

Dept. of General Surgery

Email: mitrajitsurgery@gmail.com

Mobile: +919706582392

${ }^{2}$ Senior Resident

Dept. of Pediatric Surgery

Email: kaushik1671979@rediffmail.com

Mobile: +919706194617

Gauhati Medical College and Hospital, Guwahati, Assam group. Int J Health Res Medico Leg Prae 2019 January;5(1):70-71. DOI 10.31741/ijhrmlp.v5.i1.2018.19 
operative period was uneventful and patient was discharged on the seventh post-operative day. The patient is on followup.

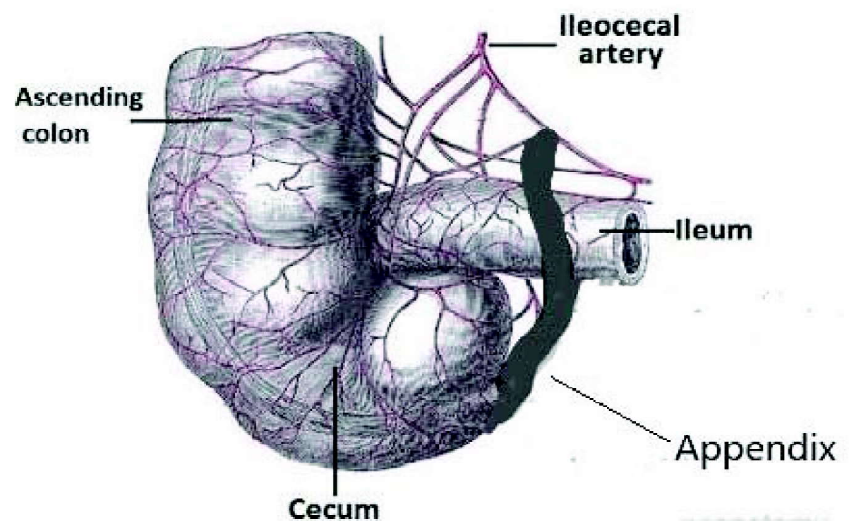

Figure 2 Inflamed appendixes is seen lying over the terminal ileum and the tip attached to the ileocecal mesentry resulting in a band like structure causing obstruction

\section{DISCUSSION}

Appendicitis causing intestinal obstruction was described as early as 1901, when Lucius Hotchkiss documented three successful surgeries for intestinal obstruction due to appendicitis. ${ }^{3}$ In 1909, Forbes Hawks divided them into mechanical, septic and a combination of the two. ${ }^{4}$ Our case was a variable of mechanical without strangulation like the case reported by Naumov in $1936 .{ }^{4}$

Clinically these patients can be classified into two types

1. Predominant features of appendicitis with some evidence of intestinal obstruction. In this group of patients, intestinal obstruction occurs during the phase of active appendicitis.

2. Patients with Acute intestinal obstruction, on laparotomy found to have appendicitis as the cause. In this group, there may or may not be a history of appendicitis. Intestinal obstruction dominates the clinical picture and may completely obscure the underlying appendicular disease. Such cases are managed accordingly by exploratory laparotomy as in our case and previously documented by Laxminarayan Bhandari. ${ }^{2}$

\section{CONCLUSION}

Intestinal obstruction due to appendicitis is of four types. Clinically and radiologically it may not be possible to differentiate these types. It may be predominantly appendicitis or predominantly intestinal obstruction. In the second group it is important to rule out appendicitis by careful re-evaluation. We found that mechanical bowel obstruction in direct connection with appendiceal inflammation is an extremely rare, but potentially life-threatening complication. A conclusive diagnosis typically has to wait until visualization during the surgical intervention. As such a case presenting with acute intestinal obstruction of unknown etiology, we need to consider appendicitis as a probable cause.

Consent: Written informed consent was obtained from the patient for publication of this case report and any accompanying images.

Conflict of interest: None Declared.

Source of funding: None Declared.

Author Disclosure: The authors do not have any disclosable interest.

\section{REFERENCES}

1. Yang AD, Lee CH. Appendico-ileal knotting resulting in closed-loop obstruction in a child. Pediatric radiology 2002 Dec 1;32(12):879-81.

2. Bhandari L, Mohandas PG. Appendicitis as a cause of intestinal strangulation: a case report and review. World J of Emergency Surgery 2009 Dec;4(1):34.

3. Hotchkiss LW. Acute intestinal obstruction following appendicitis. A report of three cases successfully operated upon. Annals of surgery 1901 Nov;34(5):660.

4. Hawkes F. The Prevention of Intestinal Obstruction Following Operation for Appendicitis. Annals of surgery 1909 Feb;49(2):192. 